\section{Riesgo de infección en pacientes con enfermedades inmunológicas y oncológicas tratados con biológicos: revisión panorámica}

\author{
MARÍA PILAR GAMBRA ${ }^{1}$, JUAN JOSÉ MONTESINOS 2 , \\ MARÍA TERESA VALENZUELA ${ }^{3}$, MARCELA ELIANA CÁRCAMO ${ }^{4}$, \\ GABRIEL CAVADA CH.,
}

\section{Risk of infections associated with the use of biological medications; a review}

Biological medications are effective for the treatment of cancer and inflammatory diseases. The aim of this review is to summarize the available evidence in systematic reviews or meta-analyses about the risk of infection in patients with cancer, arthritis, psoriasis and inflammatory bowel disease who use biological medications. We included systematic reviews or meta-analyses of controlled clinical trials and case/control studies that analyze infections during and after treatment with FDA-approved biological medications for the treatment of cancer, arthritis, inflammatory bowel disease and psoriasis, both in adults and children. The following databases were consulted: PubMed, Epistemonikos, Crochrane reviews, JIB, and Prospero. A quality guideline (AMSTAR) was applied to the selected studies. We included 26 studies. The risk of infections in patients with solid organ cancer is consistent in the literature. In psoriasis there is a risk of non-serious infections. In arthritis and other inflammatory diseases there is a risk of serious infections. In inflammatory bowel disease there is a risk for opportunistic infections. In conclusion, in patients with cancer and inflammatory diseases use biological medications entails a risk of infection. The evidence is different depending on the underlying disease of each patient.

(Rev Med Chile 2020; 148: 1155-1170)

Key words: Biological Therapy; Cancer; Inflammatory Bowel Diseases.
'Unidad de Infectología, Clínica Santa María. Santiago, Chile. ${ }^{2}$ Hospital Universitario del Río, Ecuador.

${ }^{3}$ Facultad de Medicina Universidad de los Andes. Santiago, Chile.

${ }^{4}$ Médico Veterinario, Universidad de los Andes. Santiago, Chile.

${ }^{5}$ Escuela de Salud Pública,

Universidad de Chile. Santiago,

Chile.

aBioestadístico.

Declaración de intereses: Los autores declaran no poseer conflicto de interés en la realización de este estudio.

Recibido el 15 de mayo de 2019, aceptado el 7 de mayo de 2020.

Correspondencia:

María Pilar Gambra Acle Libia 55 Depto. 202, Las Condes. Santiago, Chile.

mgambra@clinicasantamaria.cl
L

os productos biológicos son moléculas complejas usadas para prevenir, diagnosticar y tratar diferentes condiciones médicas. Hay muchos tipos de productos biológicos entre ellos proteínas terapéuticas, anticuerpos monoclonales y vacunas. Estas moléculas han sido aprobadas para su uso en un amplio rango de condiciones médicas como el cáncer, enfermedades autoinmunes, asma, infertilidad, prevención de neumonías, etc. Se ha probado su eficacia en el tratamiento de personas con enfermedades oncológicas y autoinmunes ${ }^{1-4}$.
A pesar de sus muchos beneficios, los biológicos pueden producir eventos adversos secundarios y se conocen más en la medida que su uso se expande. Algunas de estas reacciones adversas son: el síndrome de liberación de citoquinas ${ }^{5}$, alteraciones cutáneas, cáncer de piel no melanomas, colitis, diarrea y trastornos endocrinos ${ }^{6,7}$ e infecciones ${ }^{7}$.

En pacientes con cáncer de órganos sólidos y neoplasias hematológicas se ha aprobado el uso de anticuerpos monoclonales como alemtuzumab, trastuzumab, bevacizumab, cetuximab y rituximab $^{8}$. Cada anticuerpo monoclonal se uti- 
liza en monoterapia o en combinación con otros tratamientos, generalmente quimioterapia ${ }^{9}$. Los mecanismos de acción son múltiples, a modo de ejemplo trastuzumab se dirige contra el receptor 2 del factor crecimiento epidérmico humano (HER-2), tirosinkinasa implicada en el control del crecimiento y división celular. La expresión de HER-2 ocurre en algunos cánceres de mama, adenocarcinoma ovárico, pulmonar, gástrico y salival ${ }^{10}$. Bevacizumab se dirige contra el factor de crecimiento endotelial vascular ${ }^{11}$ y cetuximab se une al receptor del factor de crecimiento epidérmico (EGFR) el que desempeña un rol importante en la proliferación celular, diferenciación, apoptosis y metástasis ${ }^{12}$. Rituximab se dirige al antígeno CD20 y provoca la lisis de las líneas celulares B del linfoma no Hodking a través del complemento y la citotoxicidad dependiente de los anticuerpos ${ }^{13}$.

Food and Drug Administration (FDA) ha aprobado para enfermedades inflamatorias el uso de anti TNF $\alpha$ (infliximab, etanercept y adalimumab), antagonista de interleuquina 12 e interleuquina 23 (ustekinumab), antagonista de IL-17 (secukinumab) y un antagonista del receptor de IL 17a (ixekizumab), bloqueadores de coestimulación de células $\mathrm{T}$ (abatacept) y anti CD20 (rituximab). Estos medicamentos inhiben las vías de citoquinas de importancia crítica en el sistema inmune ${ }^{14}$.

Varios estudios han mostrado aumento en la incidencia y riesgo de infecciones serias ${ }^{15-19}$ en pacientes que usan productos biológicos. Las enfermedades respiratorias, de piel y partes blandas y genitourinario son las más frecuentes ${ }^{15}$. Este riesgo es diferente según el fármaco utilizado y es mayor cuando coexiste con altas dosis de corticoides durante la terapia ${ }^{17}$. Este riesgo puede ser dependiente del tiempo de uso, siendo mayor en los primeros 6 meses $^{20}$. El aumento de infecciones oportunistas como, por ejemplo, micobacterias tuberculosas y no tuberculosas, pueden llegar a ser importante cuando se compara su incidencia con la población general ${ }^{21-23}$. Mención especial es el riesgo de tuberculosis, que reconoce factores geográficos importantes ${ }^{24-27}$ y puede llegar a ser cuatro veces más frecuente en pacientes que usan terapia biológica comparada con aquellos que usan drogas antirreumáticas modificadoras de enfermedad (DMARDS) ${ }^{28-30}$. Herpes zoster aumenta con el uso de anti-tnf alfa en pacientes con artritis reumatoide y también aumenta este riesgo si se usa concomitantemente esteroides en altas dosis ${ }^{31}$. En pacientes con linfoma está bien establecido el aumento de riesgo de neumonía por Pneumocystis jirovecii (PCP) tratados con anti CD-20 ${ }^{32}$. El riesgo de enfermedad por citomegalovirus (CMV) en pacientes con leucemia linfática crónica (LLC) es muy alto con el uso de alemtuzumab $^{33}$. La reactivación de hepatitis B en pacientes con linfoma que usan rituximab está bien establecida ${ }^{34-36}$.

En el marco de la realización de un consenso sobre riesgo y manejo de complicaciones infecciosas en usuarios de biológicos seleccionados, al interior del comité de inmunosuprimidos de la Sociedad Chilena de Infectología, fue necesario resumir la mejor evidencia epidemiológica disponible en el menor tiempo posible, para aquellas enfermedades en las que el uso de biológicos es más frecuente en la práctica clínica según opinión de expertos nacionales en Chile, al momento de la revisión. Una revisión preliminar mostró un número importante de revisiones sistemáticas ya disponibles y decidimos realizar entonces una revisión de revisiones sistemáticas y/o metaanálisis de estudios clínicos controlados (revisión en paraguas), con la finalidad de poder analizarla.

\section{Material y Método}

\section{Tipo de estudio}

Revisión en paraguas de estudios clínicos controlados aleatorios o estudios caso/control ${ }^{37}$ (Aromataris 2015).

\section{Estrategia de búsqueda}

Se condujo una búsqueda en la literatura médica en las bases pubmed, desde 1969 hasta 31/12/2019 (anexo 1) en Epistemonikos, PROSPERO, JBI database of Systematic Review and Implementation report. Las palabras claves fueron: biologicals products, monoclonal, antibodies; infection; cancer; "Crohn's disease", ulcerative disease, arthritis, psoriasis. Los operadores booleanos AND y OR fueron utilizados en todas las bases consultadas (anexo 1).

\section{Criterios de selección}

Los artículos incluidos fueron los que cumplieron con los siguientes criterios: revisiones 
sistemáticas y/o metaanálisis de ensayos clínicos aleatorios (ECA) y de estudios de caso/control que analizan infecciones como outcome primario o secundario; posteriores al tratamiento con anticuerpos monoclonales aprobados por FDA hasta el 31/12/2019; para el tratamiento de cáncer, artritis, enfermedad inflamatoria intestinal y psoriasis; tanto en población adulta como en pediátrica; "infecciones de cualquier tipo", "infecciones serias" (asociadas a la admisión al hospital, uso de antibióticos endovenosos o muerte), "infecciones no serias" (no asociadas a muerte, hospitalización o tratamiento endovenosos), "infecciones oportunistas" (causadas por patógenos que toman ventaja de una oportunidad normalmente no disponible) e incluso infecciones específicas como tuberculosis, Herpes zoster, entre otros. El comparador fue la ausencia de intervención, placebo, otros DMARDS no biológicos. Se excluyeron metaanálisis de estudios quirúrgicos, en embarazadas y en pacientes con VIH. Los metaanálisis que incluyeron más de un grupo de enfermedad fueron atribuidos al grupo de mayor representación proporcional.

\section{Selección de revisiones sistemáticas o metaanálisis}

Dos autores (MG y JM) realizaron la selección de los artículos en base al título y resumen, revisando los objetivos y criterios de inclusión propuestos.

\section{Extracción y manejo de datos}

De los artículos seleccionados se extrajeron en forma independiente los siguientes datos: autor, revista, año de publicación, tipo de enfermedad estudiada, tipo de infección, número de estudios primarios incluidos, número de expuestos, número de controles, medicación evaluada, tipo de intervención en el grupo control, resultado examinado, tamaño del efecto (intervalo de confianza IC 95\%).

\section{Calidad de la evidencia}

Los artículos que cumplieron con criterios de selección fueron calificados utilizando una herramienta de medición de calidad para evaluar revisiones sistemáticas: "Assessing the Methodological Quality of Systematic Reviews" (AMSTAR $)^{38,39}$. La escala cuenta con 11 preguntas, cada una con cuatro posibles respuestas: sí, no, no se puede responder y no aplica. En el caso de que la respuesta fuera sí, se le asignó 1 punto y en el caso de que la respuesta fuera no, no se puede responder o no aplica, se le asignó un cero. El puntaje máximo posible fue de 11 puntos. Adicionalmente, en la misma base de datos se constató el uso de sistemas de ponderación que pudiesen suponer un sesgo de selección y limitaciones de lenguaje, ambas características no contempladas en el sistema AMSTAR.

\section{Análisis estadístico}

Se extrajeron los indicadores de riesgo de infección de cada revisión sistemática o metaanálisis: Odds Ratios (OR) o Relative Risk (RR) con su respectivo IC 95\%. De los datos obtenidos se realizó un resumen cualitativo de la evidencia.

\section{Resultados}

\section{Resultado de la búsqueda de evidencia}

Se encontraron 3912 artículos en pubmed y 191 en epistemonikos, PROSPERO, Cochrane y JIB lo que da un total de 4169 artículos identificados. Después de la revisión de títulos y resúmenes, 66 artículos fueron elegidos para su revisión en extenso. Se excluyeron 40 artículos por ser revisiones no sistemáticas, revisiones sistemáticas de estudios observacionales (cohorte o corte transversal) o revisiones sistemáticas de pacientes quirúrgicos entre otras causas. Finalmente, 26 metaanálisis fueron considerados (Figura 1): 5 en oncología, 3 en psoriasis, 13 en artritis reumatoide y 5 en enfermedad inflamatoria intestinal.

En oncología, 2 artículos hablaban de riesgo de infección en pacientes con linfoma tratados con rituximab ${ }^{40,41}$ y 3 en pacientes con tumores sólidos tratados con cetuximab y panitumumab ${ }^{42-44}$. Uno de ellos revisó tumores sólidos y cáncer hematológicos en conjunto, tratados con monoclonales ${ }^{42}$. Los comparadores fueron quimioterapia clásica, observación o radioterapia y cirugía.

De los 13 metaanálisis seleccionados en artritis reumatoide ${ }^{16,28,45-55}, 3$ incorporaron artritis reumatoide junto a otras enfermedades autoinmunes; dado que la mayor proporción de pacientes padecían artritis reumatoide, se analizaron en este grupo $^{49,53,55}$.

Las características de los artículos se muestran en las Tablas 1 y 4. 


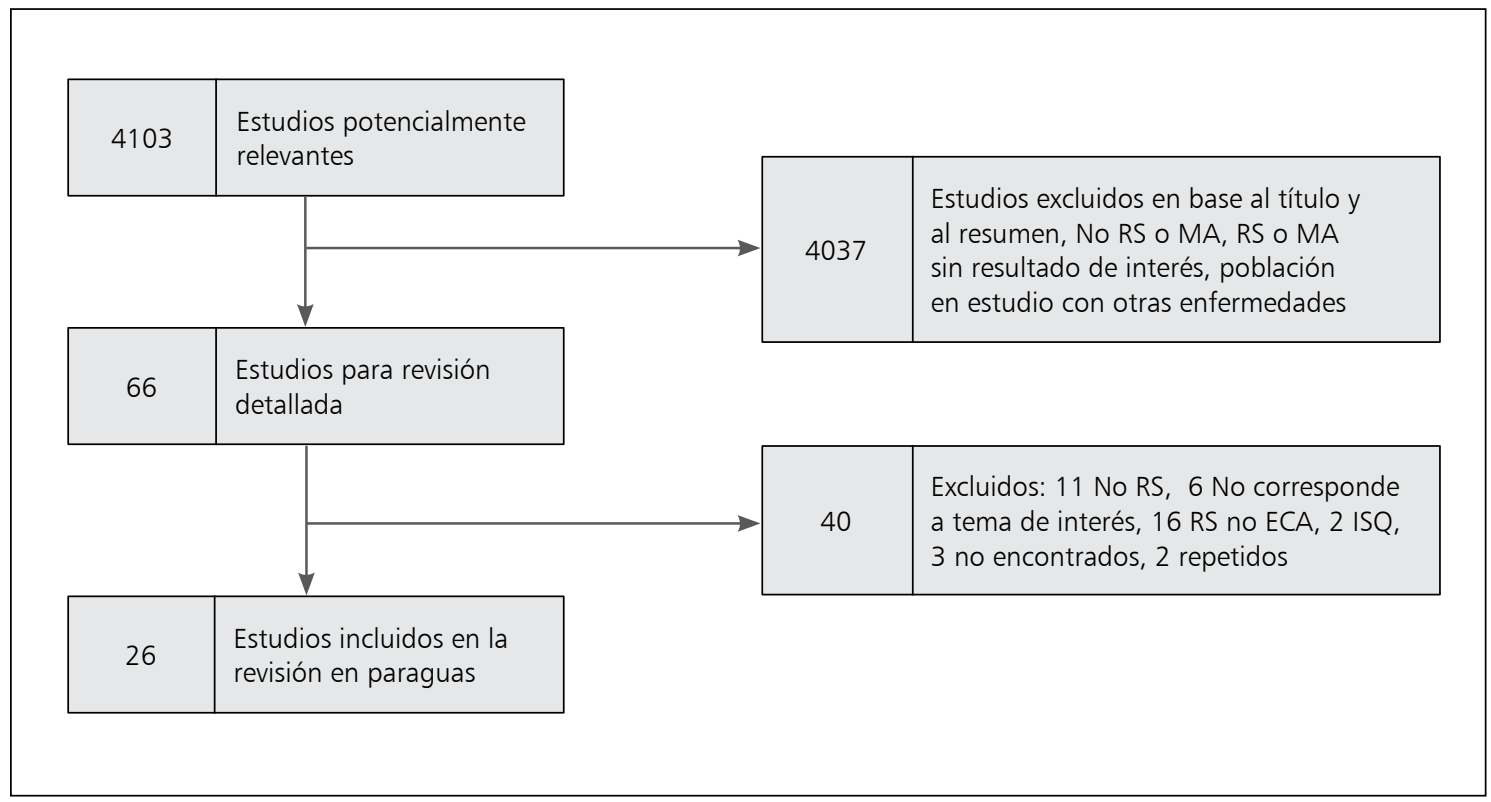

Figura 1. Resultado de la búsqueda bibliográfica.

\section{Calidad de los artículos}

No se excluyó ningún artículo por puntaje de calidad. En la Tabla 5 se muestra la calificación de los artículos seleccionados. El promedio de calidad fue 8,2 (de un rango entre 1-11), siendo los artículos en artritis reumatoide y enfermedades inflamatorias intestinal de mayor puntaje en calidad de evidencia $(8,6)$ con respecto a oncología y psoriasis ${ }^{7}$.

\section{Riesgo de infección por grupo de enfermedad}

Riesgo de infección con uso de monoclonales en pacientes con cáncer

A continuación, se describe en forma separada los estudios realizados en pacientes con linfoma y aquellos realizados en pacientes con cáncer de órganos sólidos:

- Linfoma: de los dos artículos considerados se desprende información contradictoria. Un metaanálisis no detecta riesgo de infección con rituximab: OR: 1 (IC 95\%: 0,87-1,4) ${ }^{40}$, en cambio el otro sí: OR: 2,8 (IC 95\%: 1,3-6,2) ${ }^{41}$.

- Otros cánceres (colorrectal, pulmón de células no pequeñas, cabeza y cuello, mama, urotelial, páncreas y esófago). Los 2 metaanálisis son consistentes en demostrar riesgo de infecciones y neutropenia febril en pacientes con cáncer de órganos sólidos que requieren de anticuerpos monoclonales con OR de 1,34 (IC 95\%: 1,10$1,62)$ y 1,49 (IC 95\%: 1,33-1,66) respectivamente ${ }^{43,44}$.

Riesgo de infección con el uso de monoclonales en pacientes con psoriasis

2/3 metaanálisis no reportan riesgo de infecciones serias en pacientes con psoriasis ${ }^{56-58}$. Para las infecciones no serias, Dommasch $2011^{57}$ encuentra aumento significativo para aquellos que usan anti-TNF alfa OR: 1,18 (IC 95\%: 1,05-1,33).

Riesgo de infección en el tratamiento de pacientes con artritis reumatoide

De los 13 metaanálisis, 6 analizaron infecciones serias ${ }^{28,46,50,59,60}, 3$ infecciones de cualquier tipo $^{16,47,54}, 2$ tuberculosis ${ }^{49,55}$, uno Herpes zoster $^{52}$ $\mathrm{y}$ uno infecciones no serias ${ }^{53}$. En infecciones no serias el riesgo es significativamente menor para etanercept cuando se compara con adalimumab e infliximab. El riesgo de Herpes zoster fue 1,61 (IC 95\%: 1,16-2,23) ${ }^{52}$. Los estudios de Souto ${ }^{49}$ : OR: 1,92 (IC 95\%: 0,91-4,83) y Capogrosso ${ }^{55}$ : OR: 2,47 (IC 95\%: 0,64-9,56), no muestran riesgo de tuberculosis con el uso de biológicos 


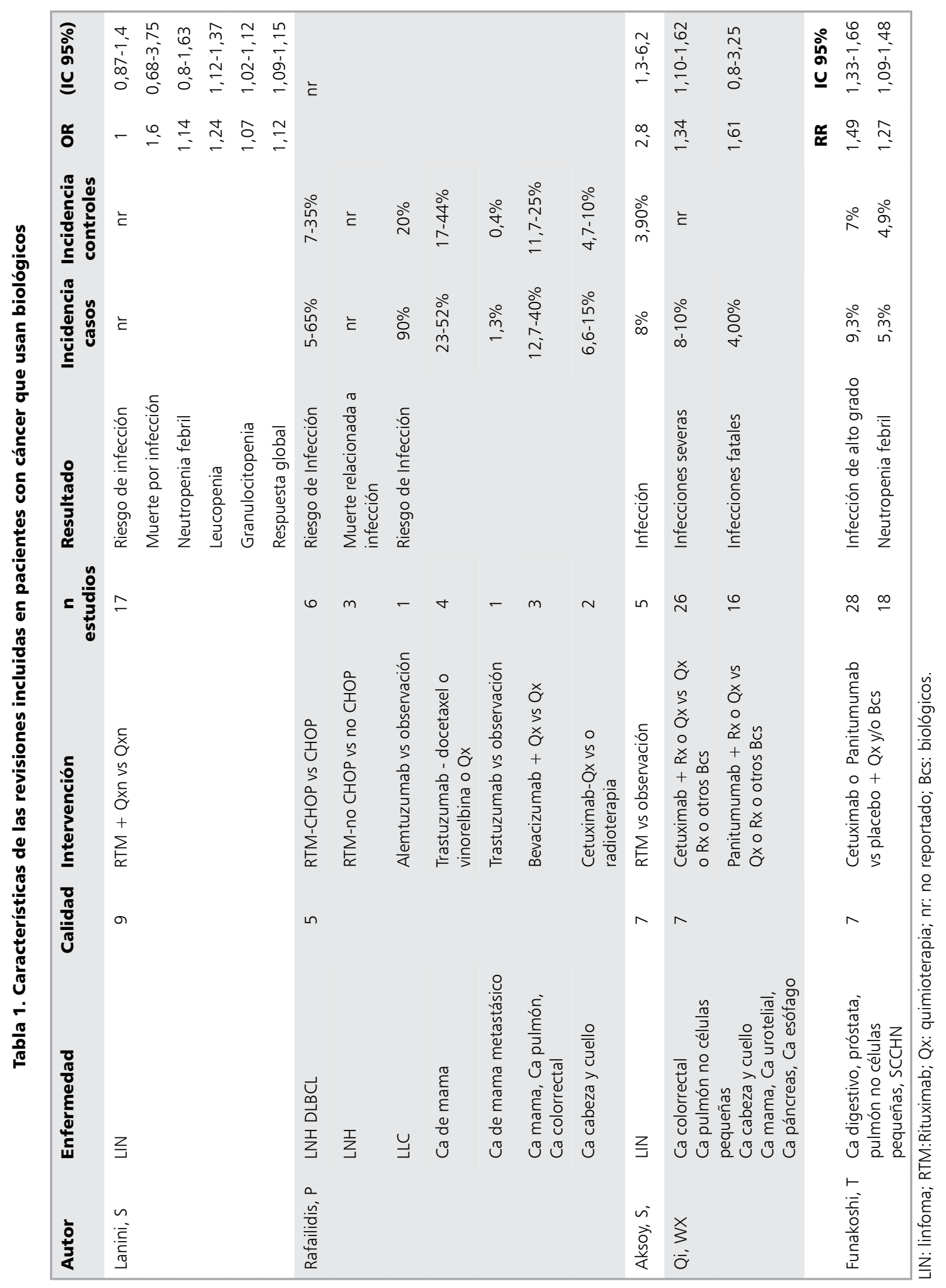




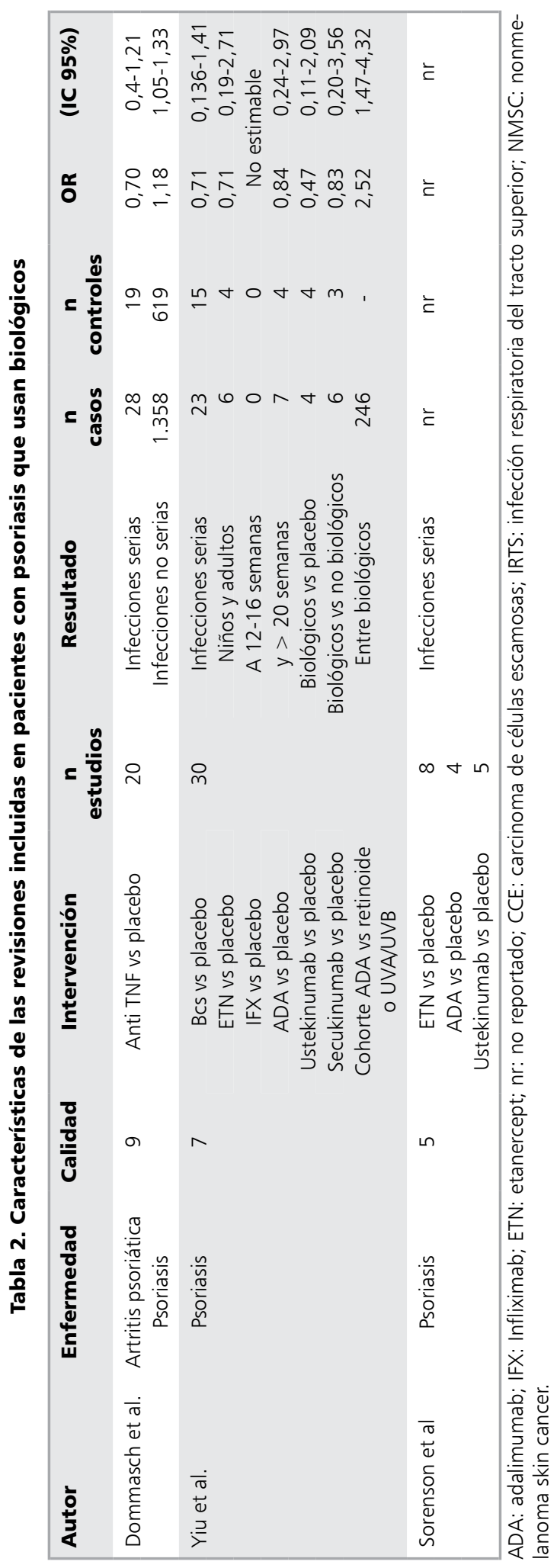

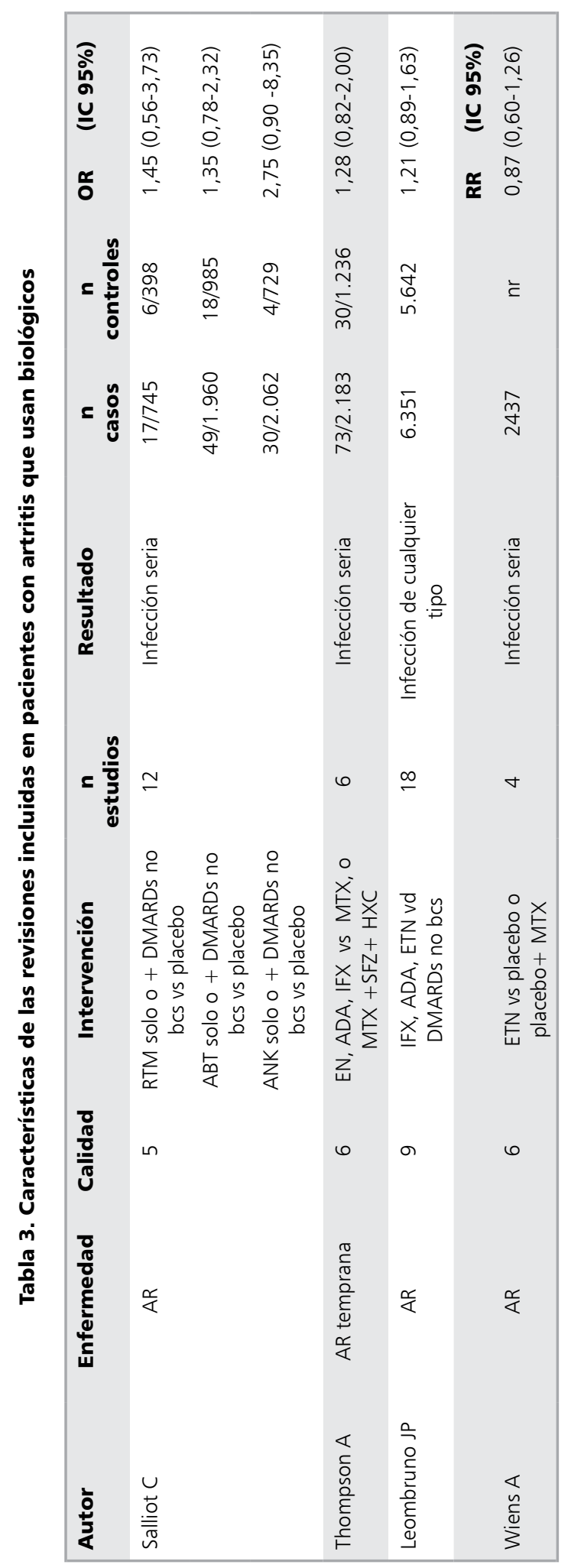




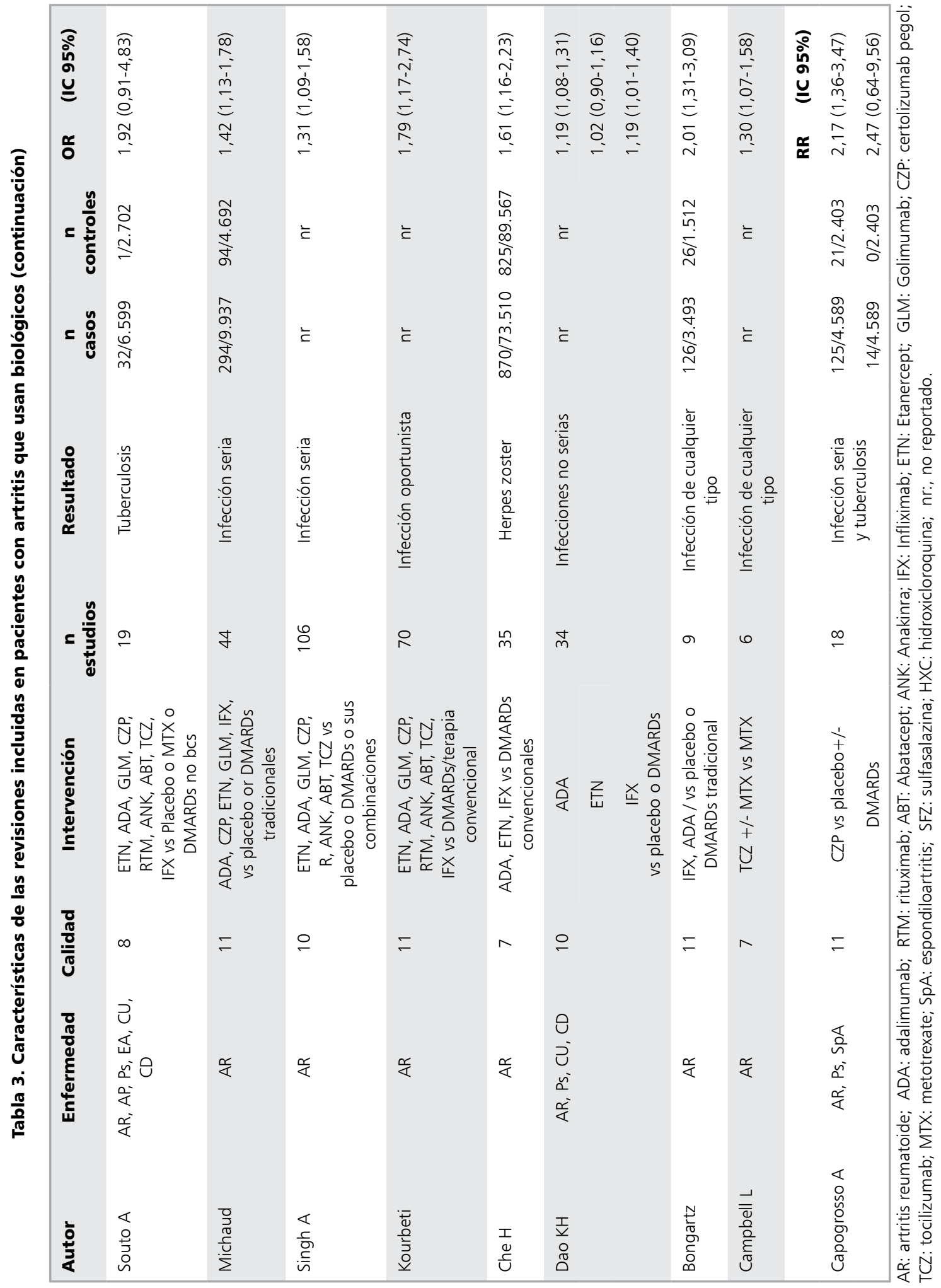




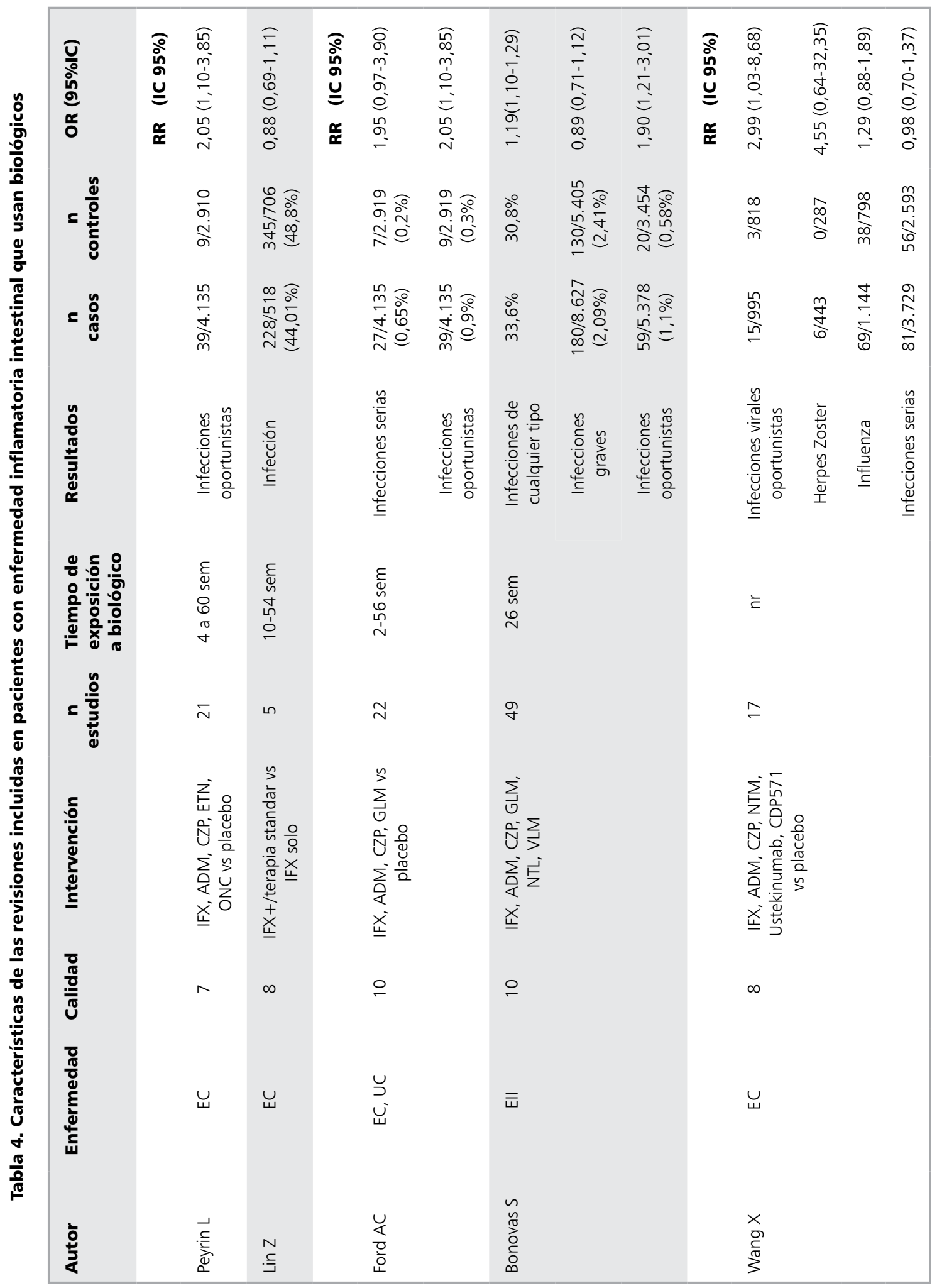




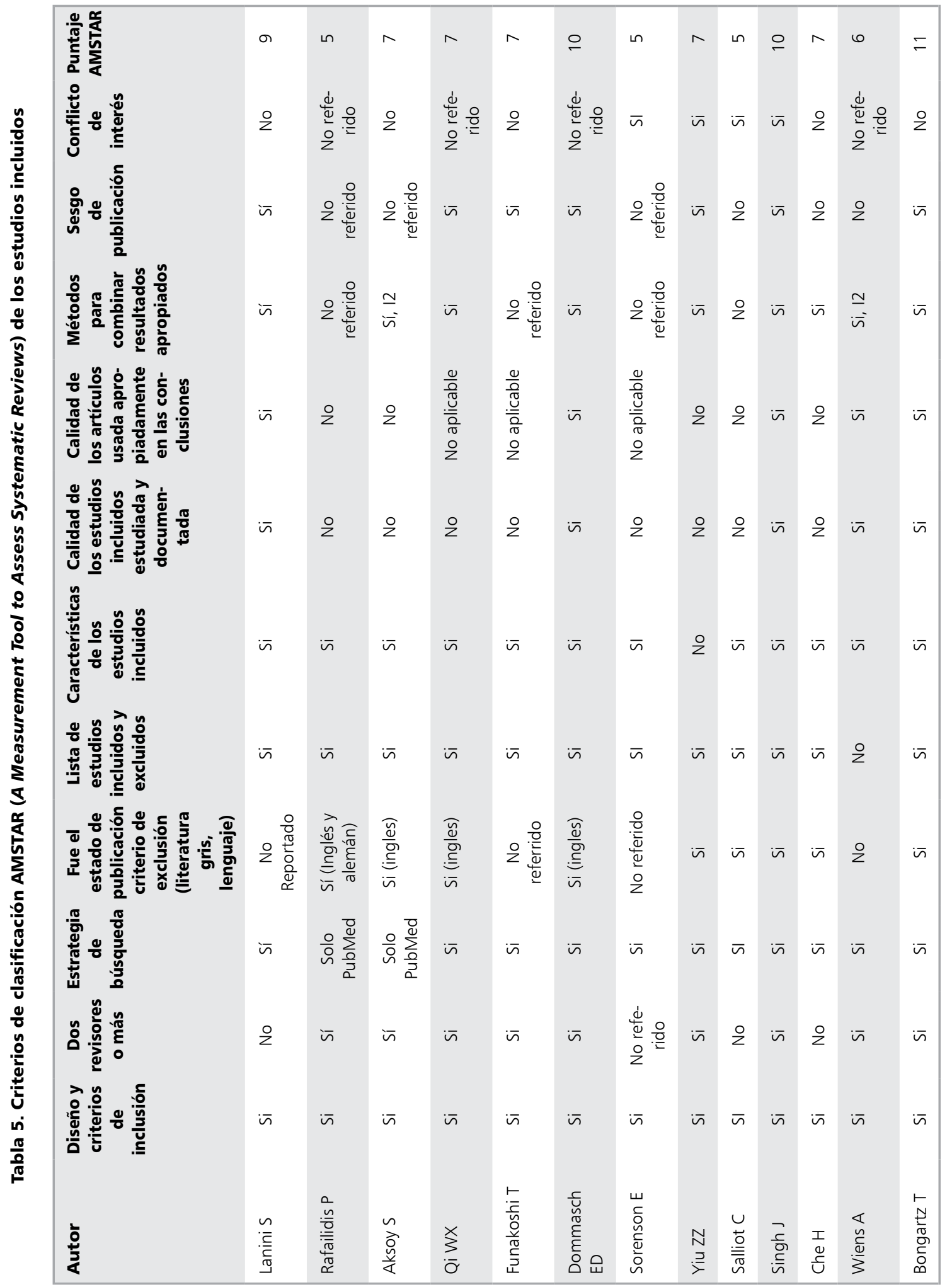




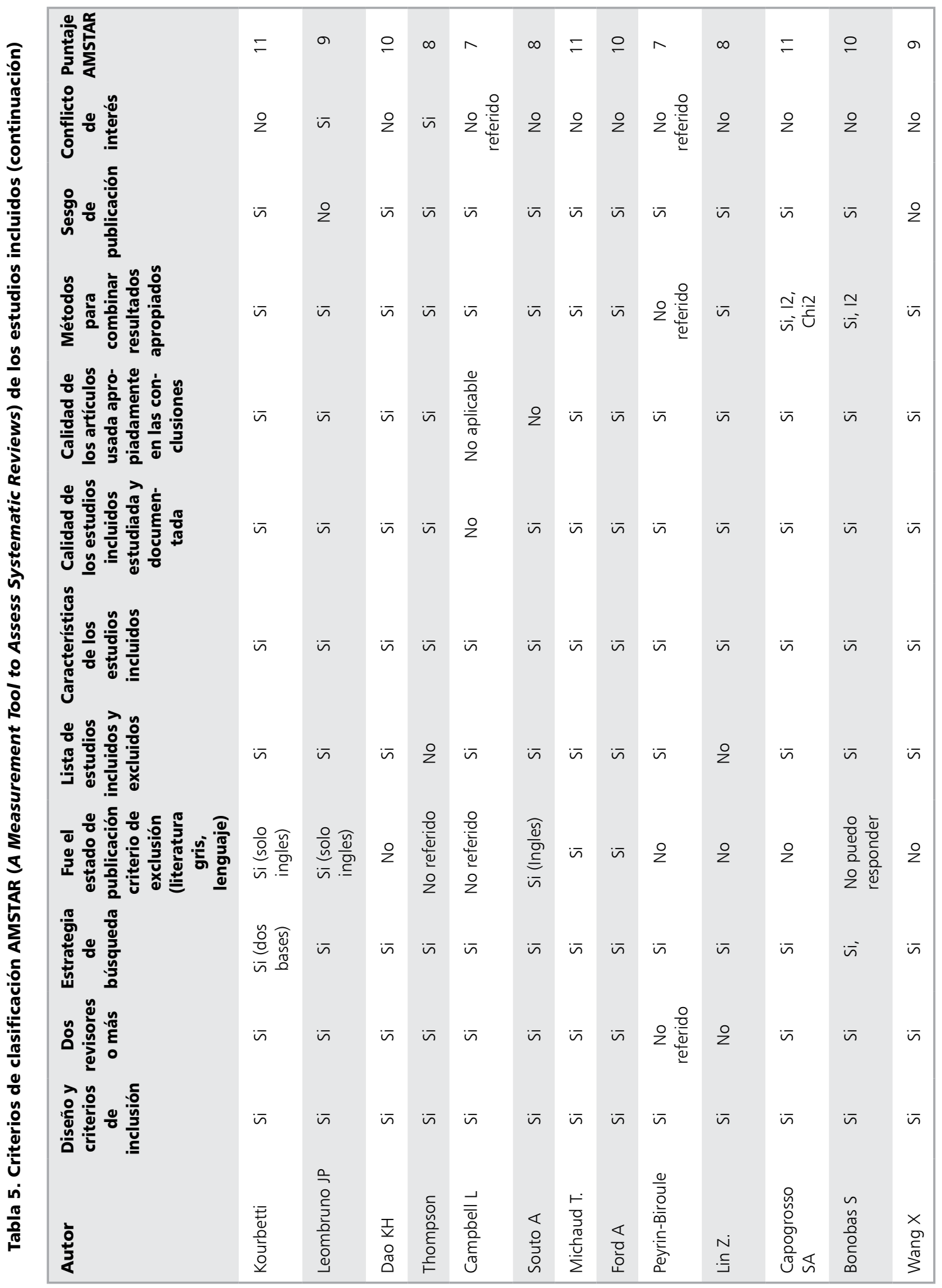


en pacientes con enfermedades inflamatorias, mayoritariamente con artritis reumatoide. Con mayores dosis y biológicos específicos, el riesgo de infección seria aumenta: anakinra con OR: 9,63 (IC 95\%: 1,31-70,91) ${ }^{59} \mathrm{y}$ tocilizumab OR: 1,30; (IC 95\%: $1,07-1,58)^{54}$.

Riesgo de infección con monoclonales en pacientes con enfermedad inflamatoria intestinal

En todos los metaanálisis incluidos ${ }^{61-65}$ no se observó riesgo significativo de infecciones serias. Ford ${ }^{29}$ y Bonovas ${ }^{64}$ demostraron una duplicación del riesgo de infecciones oportunistas en estos pacientes OR: 2,05 (IC 95\%: 1,10-3,85) y OR: 1,90 (IC 95\%: 1,21-3,01) respectivamente.

\section{Discusión}

Esta es la primera revisión sistemática de revisiones sistemáticas y/o metaanálisis que resume la evidencia disponible del riesgo de infección en usuarios de biológicos por grupo de enfermedad.

En pacientes con linfoma los resultados de ambos metaanálisis son contradictorios mientras que en tumores sólidos los resultados apuntan claramente a que existe riesgo de infección en pacientes que usan biológicos. Este hecho es posible de explicar por la sensibilización de los equipos de oncología con el uso sistemático de quimioprofilaxis en pacientes con linfomas, lo que no ocurre en pacientes con cáncer de órganos sólidos ${ }^{66}$. Puede ser favorable incorporar quimioprofilaxis a paciente con cáncer de órganos sólidos que usan biológicos y esto debiera ser materia de estudio en el futuro. En pacientes con psoriasis no parece haber efecto de biológicos en determinar el riesgo de infecciones serias, pero sí de infecciones no serias. Esto podría estar relacionado a que la mayoría de pacientes con psoriasis, no requieren de drogas sistémicas. En artritis no se pudo mostrar riesgo de tuberculosis ${ }^{49,55}$. Presumimos que ECA no son sensibles para determinar reactivación de tuberculosis latente ya que en estudios observacionales y reporte de eventos adversos el aumento de la incidencia ha sido bien demostrado ${ }^{24,26,68}$.

En enfermedad inflamatoria intestinal el riesgo de infecciones serias no es significativo en los estudios analizados ${ }^{29,61,70}$, pero sí de infecciones oportunistas. De estas, las más frecuente es tuberculosis y ello indica que mejoras en el screening de tuberculosis latente y tratamiento precoz podría impactar el riesgo de infecciones oportunistas $^{71}$.

Este análisis tiene varias limitaciones la principal de todas es que el resultado de cada estudio primario es con frecuencia un objetivo secundario dentro de un estudio de seguridad. La información es robusta, pero limitada. Los ECA permiten explorar el riesgo de infección, pero pueden ser débiles en infecciones como tuberculosis que requieren más tiempo de exposición farmacológica para manifestarse. Otra limitación, es que los estudios son heterogéneos en la severidad de la enfermedad, tipo de biológicos estudiados, comparadores y duración de terapias, definiciones de infecciones, lo que puede impedir conclusiones definitivas. Algunos estudios analizan más de un resultado (Ej.: infecciones serias y tuberculosis), lo que es difícil de interpretar y otros estudios fueron realizados en el conjunto de enfermedades autoinmunes, lo que podría cuestionar los hallazgos encontrados para el grupo de artritis. Los estudios secundarios tienen el potencial sesgo de publicación, específicamente de los estudios que muestran un efecto, ya que son más fáciles de promover que aquellos que no muestran efecto. Por último, los resultados encontrados no pueden extrapolarse a población pediátrica dado que la totalidad de los estudios seleccionados fueron de población adulta. Tampoco se ha representado la población de pacientes con artritis y enfermedad inflamatoria intestinal que requieren cirugía durante el uso de biológicos, ni embarazadas, ni pacientes HIV.

En 2011, Singh realizó un network metaanálisis con el supuesto de que las condiciones subyacentes no determinan mayor riesgo de padecer enfermedades infecciosas ${ }^{19}$. Este estudio supone lo contrario y es el hecho de que el riesgo es diferente dependiendo de la enfermedad de base. El perfil de riesgo de estos pacientes puede ser significativamente alterado por el uso de otras drogas inmunosupresoras concomitantes. Son muchos los factores que determinan riesgo de infección: el tipo de biológico, diversos mecanismos de acción, dosis y duración de la terapia, el riesgo es diferente en el tiempo, tiempo de uso y tiempo de seguimiento. Las enfermedades como la tuberculosis reconocen, además, diferencia geográfica.

En base a lo anteriormente expuesto, la evidencia encontrada debe generar cambios en las 
conductas clínicas en pacientes que usan biológicos. El tratante debe estar atento a la presencia de síntomas y signos que pueden indicar infección serias, no serias y oportunistas, para intervenir precozmente en su tratamiento.

Es necesario continuar enriqueciendo el conocimiento y ampliar los resultados de este estudio, incorporando los resultados de estudios observa- cionales y registros clínicos, que permitan valorar el riesgo en el tiempo, así como en el mundo real.

Agradecimientos: Comité de inmunocomprometidos de la Sociedad Chilena de Infectología y Universidad de los Andes (Magíster de Epidemiología, Departamento de Salud Pública y Epidemiología).

\section{Anexo. Estrategia de búsqueda}

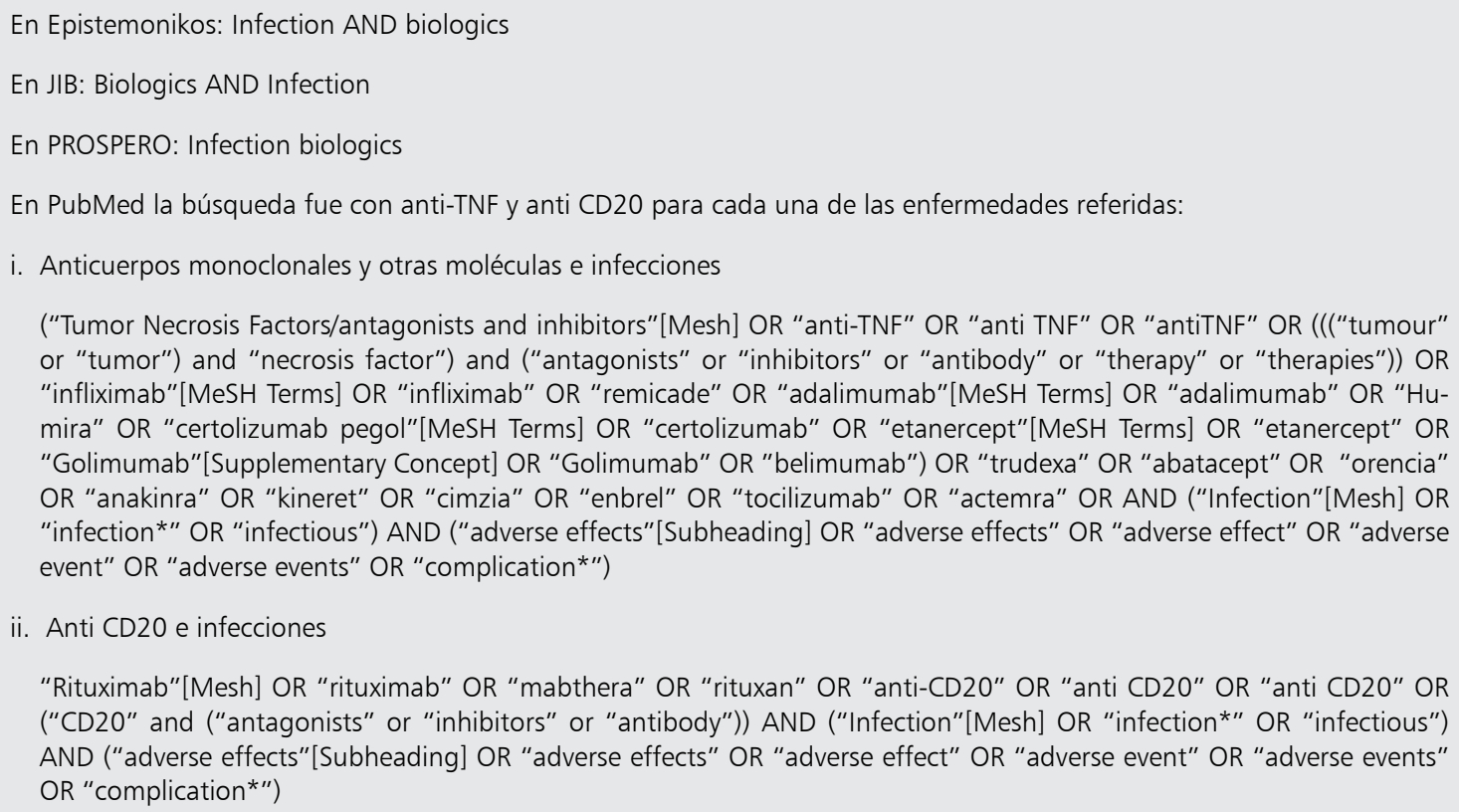
or "tumor") and "necrosis factor") and ("antagonists" or "inhibitors" or "antibody" or "therapy" or "therapies")) OR "infliximab"[MeSH Terms] OR "infliximab" OR "remicade" OR "adalimumab"[MeSH Terms] OR "adalimumab" OR "Humira" OR "certolizumab pegol"[MeSH Terms] OR "certolizumab" OR "etanercept"[MeSH Terms] OR "etanercept" OR "Golimumab"[Supplementary Concept] OR "Golimumab" OR "belimumab") OR "trudexa" OR "abatacept" OR "orencia" OR "anakinra" OR "kineret" OR "cimzia" OR "enbrel" OR "tocilizumab" OR "actemra" OR AND ("Infection"[Mesh] OR "infection*" OR "infectious") AND ("adverse effects"[Subheading] OR "adverse effects" OR "adverse effect" OR "adverse event" OR "adverse events" OR "complication*")

ii. Anti CD20 e infecciones

"Rituximab"[Mesh] OR "rituximab" OR "mabthera" OR "rituxan" OR "anti-CD20" OR "anti CD20" OR "anti CD20" OR ("CD20" and ("antagonists" or "inhibitors" or "antibody")) AND ("Infection"[Mesh] OR "infection*" OR "infectious") AND ("adverse effects"[Subheading] OR "adverse effects" OR "adverse effect" OR "adverse event" OR "adverse events" OR "complication*")

iii. Sin restricción de idioma

Filtro en PubMed revisiones sistemáticas y metaanálisis en PubMed y para revisiones sistemáticas en epistemonikos

Fecha de la búsqueda en PubMed desde 1970 hasta el 31/12/2019 y en Epistemonikos los últimos 10 años, búsqueda realizada el 31/12/2019.

Filtro de página web en humanos.

\section{Referencias}

1. Singh S, Kumar NK, Dwiwedi P, Charan J, Kaur R, Sidhu P, et al. Monoclonal Antibodies: A Review. Curr Clin Pharmacol 2018; 13 (2): 85-99.

2. Bilal J, Berlinberg A, Bhattacharjee S, Trost J, Riaz IB, Kurtzman DJB. A systematic review and meta-analysis of the efficacy and safety of the interleukin (IL)-12/23 and IL-17 inhibitors ustekinumab, secukinumab, ixeki- zumab, brodalumab, guselkumab and tildrakizumab for the treatment of moderate to severe plaque psoriasis. J Dermatolog Treat 2018; 29 (6): 569-78.

3. Stadler N, Shang A, Bosch F, Briggs A, Goede V, Berthier A, et al. A Systematic Review and Network Meta-Analysis to Evaluate the Comparative Efficacy of Interventions for Unfit Patients with Chronic Lymphocytic Leukemia. Adv Ther 2016; 33 (10): 1814-30.

4. Ma K, Li L, Liu C, Zhou L, Zhou X. Efficacy and safety 
of various anti-rheumatic treatments for patients with rheumatoid arthritis: a network meta-analysis. Arch Med Sci 2019; 15 (1): 33-54.

5. French JB, Bonacini M, Ghabril M, Foureau D, Bonkovsky HL. Hepatotoxicity Associated with the Use of Anti-TNF- $\alpha$ Agents. Drug Saf 2016; 39 (3): 199-208.

6. Kroschinsky F, Stölzel F, von Bonin S, Beutel G, Kochanek M, Kiehl M, et al. New drugs, new toxicities: severe side effects of modern targeted and immunotherapy of cancer and their management. Crit Care 2017; 21 (1): 89.

7. Murdaca G, Negrini S, Pellecchio M, Greco M, Schiavi $\mathrm{C}$, Giusti F, et al. Update upon the infection risk in patients receiving TNF alpha inhibitors. Expert Opin Drug Saf 2019: 1-11.

8. Adams GP, Weiner LM. Monoclonal antibody therapy of cancer. Nat Biotechnol 2005; 23 (9): 1147-57.

9. Prica A, Baldassarre F, Hicks LK, Imrie K, Kouroukis T, Cheung M, et al. Rituximab in Lymphoma and Chronic Lymphocytic Leukaemia: A Practice Guideline. Clin Oncol (R Coll Radiol) 2017; 29 (1): e13-e28.

10. Rinnerthaler G, Gampenrieder SP, Greil R. ASCO 2018 highlights: metastatic breast cancer. Memo 2018; 11 (4): 276-9.

11. Tamura R, Tanaka T, Miyake K, Yoshida K, Sasaki H. Bevacizumab for malignant gliomas: current indications, mechanisms of action and resistance, and markers of response. Brain Tumor Pathol 2017; 34 (2): 62-77.

12. Blaszczak W, Barczak W, Wegner A, Golusinski W, Suchorska WM. Clinical value of monoclonal antibodies and tyrosine kinase inhibitors in the treatment of head and neck squamous cell carcinoma. Med Oncol 2017; 34 (4): 60 .

13. Creus N, Massó J, Codina C, Ribas J. Anticuerpos monoclonales en Oncología. Farmacia Hospitalaria 2002; 26 (1): 28-43.

14. Serio I, Tovoli F. Rheumatoid arthritis: new monoclonal antibodies. Drugs Today (Barc) 2018; 54 (3): 219-30.

15. Rutherford AI, Subesinghe S, Hyrich KL, Galloway JB. Serious infection across biologic-treated patients with rheumatoid arthritis: results from the British Society for Rheumatology Biologics Register for Rheumatoid Arthritis. Ann Rheum Dis 2018; 77 (6): 905-10.

16. Bongartz T, Sutton AJ, Sweeting MJ, Buchan I, Matteson EL, Montori V. Anti-TNF antibody therapy in rheumatoid arthritis and the risk of serious infections and malignancies: systematic review and meta-analysis of rare harmful effects in randomized controlled trials. JAMA 2006; 295 (19): 2275-85.

17. Grijalva CG, Chen L, Delzell E, Baddley JW, Beukelman $\mathrm{T}$, Winthrop KL, et al. Initiation of tumor necrosis factor- $\alpha$ antagonists and the risk of hospitalization for infection in patients with autoimmune diseases. JAMA. 2011; 306 (21): 2331-9.

18. Becker I, Horneff G. Risk of Serious Infection in Juvenile Idiopathic Arthritis Patients Associated With Tumor Necrosis Factor Inhibitors and Disease Activity in the German Biologics in Pediatric Rheumatology Registry. Arthritis Care Res (Hoboken) 2017; 69 (4): 552-60.

19. Singh JA, Wells GA, Christensen R, Tanjong Ghogomu E, Maxwell L, Macdonald JK, et al. Adverse effects of biologics: a network meta-analysis and Cochrane overview. Cochrane Database Syst Rev 2011 (2): CD008794.

20. Galloway JB, Hyrich KL, Mercer LK, Dixon WG, Fu B, Ustianowski AP, et al. Anti-TNF therapy is associated with an increased risk of serious infections in patients with rheumatoid arthritis especially in the first 6 months of treatment: updated results from the British Society for Rheumatology Biologics Register with special emphasis on risks in the elderly. Rheumatology (Oxford) 2011; 50 (1): 124-31.

21. Salmon-Ceron D, Tubach F, Lortholary O, Chosidow O, Bretagne S, Nicolas N, et al. Drug-specific risk of non-tuberculosis opportunistic infections in patients receiving anti-TNF therapy reported to the 3 -year prospective French RATIO registry. Ann Rheum Dis 2011; 70 (4): 616-23.

22. Lanternier F, Tubach F, Ravaud P, Salmon D, Dellamonica $\mathrm{P}$, Bretagne $\mathrm{S}$, et al. Incidence and risk factors of Legionella pneumophila pneumonia during anti-tumor necrosis factor therapy: a prospective French study. Chest 2013; 144 (3): 990-8.

23. Tubach F, Salmon D, Ravaud P, Allanore Y, Goupille $\mathrm{P}$, Bréban $\mathrm{M}$, et al. Risk of tuberculosis is higher with anti-tumor necrosis factor monoclonal antibody therapy than with soluble tumor necrosis factor receptor therapy: The three-year prospective French Research Axed on Tolerance of Biotherapies registry. Arthritis Rheum 2009; 60 (7): 1884-94.

24. Winthrop KL, Novosad SA, Baddley JW, Calabrese L, Chiller T, Polgreen P, et al. Opportunistic infections and biologic therapies in immune-mediated inflammatory diseases: consensus recommendations for infection reporting during clinical trials and postmarketing surveillance. Ann Rheum Dis 2015; 74 (12): 2107-16.

25. Arkema EV, Jonsson J, Baecklund E, Bruchfeld J, Feltelius N, Askling J, et al. Are patients with rheumatoid arthritis still at an increased risk of tuberculosis and what is the role of biological treatments? Ann Rheum Dis 2015; 74 (6): 1212-7.

26. Gómez-Reino JJ, Carmona L, Angel Descalzo M, Group B. Risk of tuberculosis in patients treated with tumor 
necrosis factor antagonists due to incomplete prevention of reactivation of latent infection. Arthritis Rheum 2007; 57 (5): 756-61.

27. Nacci F, Matucci-Cerinic M. Tuberculosis and other infections in the anti-tumour necrosis factor-alpha (anti-TNF- $\alpha$ ) era. Best Pract Res Clin Rheumatol. 2011; 25 (3): 375-88.

28. Singh JA, Cameron C, Noorbaloochi S, Cullis T, Tucker $\mathrm{M}$, Christensen R, et al. Risk of serious infection in biological treatment of patients with rheumatoid arthritis: a systematic review and meta-analysis. Lancet 2015; 386 (9990): 258-65.

29. Ford AC, Peyrin-Biroulet L. Opportunistic infections with anti-tumor necrosis factor-alpha therapy in inflammatory bowel disease: meta-analysis of randomized controlled trials. Am J Gastroenterol 2013; 108 (8): 1268-76.

30. Minozzi S, Bonovas S, Lytras T, Pecoraro V, González-Lorenzo M, Bastiampillai AJ, et al. Risk of infections using anti-TNF agents in rheumatoid arthritis, psoriatic arthritis, and ankylosing spondylitis: a systematic review and meta-analysis. Expert Opin Drug Saf 2016; 15 (sup1): 11-34.

31. Strangfeld A, Listing J, Herzer P, Liebhaber A, Rockwitz $\mathrm{K}$, Richter $\mathrm{C}$, et al. Risk of herpes zoster in patients with rheumatoid arthritis treated with anti-TNF-alpha agents. JAMA 2009; 301 (7): 737-44.

32. Jiang X, Mei X, Feng D, Wang X. Prophylaxis and Treatment of Pneumocystis jiroveci Pneumonia in Lymphoma Patients Subjected to Rituximab-Contained Therapy: A Systemic Review and Meta-Analysis. PLoS One 2015; 10 (4): e0122171.

33. Skoetz N, Bauer K, Elter T, Monsef I, Roloff V, Hallek $\mathrm{M}$, et al. Alemtuzumab for patients with chronic lymphocytic leukaemia. The Cochrane Library. 2012.

34. Ye H, Zhang XW, Mu R, Fang LK, Gu JR, Lin J, et al. Anti-TNF therapy in patients with HBV infection-analysis of 87 patients with inflammatory arthritis. Clin Rheumatol 2014; 33 (1): 119-23.

35. Tang Z, Li X, Wu S, Liu Y, Qiao Y, Xu D, et al. Risk of hepatitis $B$ reactivation in HBsAg-negative/HBcAb-positive patients with undetectable serum HBV DNA after treatment with rituximab for lymphoma: a meta-analysis. Hepatol Int. 2017; 11 (5): 429-33.

36. Perrillo RP, Gish R, Falck-Ytter YT. American Gastroenterological Association Institute technical review on prevention and treatment of hepatitis B virus reactivation during immunosuppressive drug therapy. Gastroenterology 2015; 148 (1): 221-44.e3.

37. Grant MJ, Booth A. A typology of reviews: an analysis of 14 review types and associated methodologies. Health
Info Libr J 2009; 26 (2): 91-108.

38. Shea BJ, Hamel C, Wells GA, Bouter LM, Kristjansson E, Grimshaw J, et al. AMSTAR is a reliable and valid measurement tool to assess the methodological quality of systematic reviews. J Clin Epidemiol 2009; 62 (10): 1013-20.

39. Shea BJ, Grimshaw JM, Wells GA, Boers M, Andersson N, Hamel C, et al. Development of AMSTAR: a measurement tool to assess the methodological quality of systematic reviews. BMC Med Res Methodol. 2007; 7: 10.

40. Lanini S, Molloy AC, Fine PE, Prentice AG, Ippolito G, Kibbler CC. Risk of infection in patients with lymphoma receiving rituximab: systematic review and meta-analysis. BMC Med 2011; 9: 36.

41. Aksoy S, Dizdar O, Hayran M, Harputluoglu H. Infectious complications of rituximab in patients with lymphoma during maintenance therapy: a systematic review and meta-analysis. Leuk Lymphoma. 2009; 50 (3): 357-65.

42. Rafailidis PI, Kakisi OK, Vardakas K, Falagas ME. Infectious complications of monoclonal antibodies used in cancer therapy: a systematic review of the evidence from randomized controlled trials. Cancer 2007; 109 (11): 2182-9.

43. Qi WX, Fu S, Zhang Q, Guo XM. Incidence and risk of severe infections associated with anti-epidermal growth factor receptor monoclonal antibodies in cancer patients: a systematic review and meta-analysis. BMC Med 2014; 12: 203.

44. Funakoshi T, Suzuki M, Tamura K. Infectious complications in cancer patients treated with anti-EGFR monoclonal antibodies cetuximab and panitumumab: a systematic review and meta-analysis. Cancer Treat Rev 2014; 40 (10): 1221-9.

45. Salliot C, Dougados M, Gossec L. Risk of serious infections during rituximab, abatacept and anakinra treatments for rheumatoid arthritis: meta-analyses of randomised placebo-controlled trials. Ann Rheum Dis.2009; 68 (1): 25-32.

46. Thompson AE, Rieder SW, Pope JE. Tumor necrosis factor therapy and the risk of serious infection and malignancy in patients with early rheumatoid arthritis: a meta-analysis of randomized controlled trials. Arthritis Rheum.2011; 63 (6): 1479-85.

47. Leombruno JP, Einarson TR, Keystone EC. The safety of anti-tumour necrosis factor treatments in rheumatoid arthritis: meta and exposure-adjusted pooled analyses of serious adverse events. Ann Rheum Dis 2009; 68 (7): 1136-45.

48. Wiens A, Venson R, Correr CJ, Otuki MF, Pontarolo R. 
Meta-analysis of the efficacy and safety of adalimumab, etanercept, and infliximab for the treatment of rheumatoid arthritis. Pharmacotherapy 2010; 30 (4): 339-53.

49. Souto A, Maneiro JR, Salgado E, Carmona L, Gómez-Reino JJ. Risk of tuberculosis in patients with chronic immune-mediated inflammatory diseases treated with biologics and tofacitinib: a systematic review and meta-analysis of randomized controlled trials and long-term extension studies. Rheumatology (Oxford) 2014; 53 (10): 1872-85.

50. Michaud TL, Rho YH, Shamliyan T, Kuntz KM, Choi HK. The comparative safety of tumor necrosis factor inhibitors in rheumatoid arthritis: a meta-analysis update of 44 trials. Am J Med. 2014; 127 (12): 1208-32.

51. Kourbeti IS, Ziakas PD, Mylonakis E. Biologic therapies in rheumatoid arthritis and the risk of opportunistic infections: a meta-analysis. Clin Infect Dis 2014; 58 (12): 1649-57.

52. Che H, Lukas C, Morel J, Combe B. Risk of herpes/ herpes zoster during anti-tumor necrosis factor therapy in patients with rheumatoid arthritis. Systematic review and meta-analysis. Joint Bone Spine 2014; 81 (3): 21521.

53. Dao KH, Herbert M, Habal N, Cush JJ. Nonserious infections: should there be cause for serious concerns? Rheum Dis Clin North Am 2012; 38 (4): 707-25.

54. Campbell L, Chen C, Bhagat SS, Parker RA, Ostor AJ. Risk of adverse events including serious infections in rheumatoid arthritis patients treated with tocilizumab: a systematic literature review and meta-analysis of randomized controlled trials. Rheumatology (Oxford) 2011; 50 (3): 552-62.

55. Capogrosso Sansone A, Mantarro S, Tuccori M, Ruggiero E, Montagnani S, Convertino I, et al. Safety Profile of Certolizumab Pegol in Patients with Immune-Mediated Inflammatory Diseases: A Systematic Review and Meta-Analysis. Drug Saf 2015; 38 (10): 869-88.

56. Yiu ZZ, Exton LS, Jabbar-López Z, Mohd Mustapa MF, Samarasekera EJ, Burden AD, et al. Risk of Serious Infections in Patients with Psoriasis on Biologic Therapies: A Systematic Review and Meta-Analysis. J Invest Dermatol 2016; 136 (8): 1584-91.

57. Dommasch ED, Abuabara K, Shin DB, Nguyen J, Troxel $\mathrm{AB}$, Gelfand JM. The risk of infection and malignancy with tumor necrosis factor antagonists in adults with psoriatic disease: a systematic review and meta-analysis of randomized controlled trials. J Am Acad Dermatol 2011; 64 (6): 1035-50.

58. Sorenson E, Koo J. Evidence-based adverse effects of biologic agents in the treatment of moderate-to-severe psoriasis: Providing clarity to an opaque topic. J Derma- tolog Treat 2015; 26 (6): 493-501.

59. Salliot C, Dougados M, Gossec L. Risk of serious infections during rituximab, abatacept and anakinra treatments for rheumatoid arthritis: meta-analyses of randomised placebo-controlled trials. Annals of the rheumatic diseases 2009; 68 (1): 25-32.

60. Wiens A, Correr CJ, Venson R, Otuki MF, Pontarolo R. A systematic review and meta-analysis of the efficacy and safety of adalimumab for treating rheumatoid arthritis. Rheumatol Int 2010; 30 (8): 1063-70.

61. Peyrin-Biroulet L, Deltenre P, de Suray N, Branche J, Sandborn WJ, Colombel JF. Efficacy and safety of tumor necrosis factor antagonists in Crohn's disease: meta-analysis of placebo-controlled trials. Clin Gastroenterol Hepatol 2008; 6 (6): 644-53.

62. Lin Z, Bai Y, Zheng P. Meta-analysis: efficacy and safety of combination therapy of infliximab and immunosuppressives for Crohn's disease. Eur J Gastroenterol Hepatol 2011; 23 (12): 1100-10.

63. Ford AC, Sandborn WJ, Khan KJ, Hanauer SB, Talley NJ, Moayyedi P. Efficacy of biological therapies in inflammatory bowel disease: systematic review and meta-analysis. The American journal of gastroenterology 2011; 106 (4): 644-59.

64. Bonovas S, Pantavou K, Evripidou D, Bastiampillai AJ, Nikolopoulos GK, Peyrin-Biroulet L, et al. Safety of biological therapies in ulcerative colitis: An umbrella review of meta-analyses. Best Pract Res Clin Gastroenterol. 2018; 32-33: 43-7.

65. Wang X, Zhou F, Zhao J, Zhou R, Huang M, Li J, et al. Elevated risk of opportunistic viral infection in patients with Crohn's disease during biological therapies: a meta analysis of randomized controlled trials. Eur J Clin Pharmacol 2013; 69 (11): 1891-9.

66. Taplitz RA, Kennedy EB, Bow EJ, Crews J, Gleason C, Hawley DK, et al. Antimicrobial Prophylaxis for Adult Patients With Cancer-Related Immunosuppression: ASCO and IDSA Clinical Practice Guideline Update. J Clin Oncol. 2018: JCO1800374.

67. Wang L, Chen YZ, Shi D, Shi XY, Zou Z, Zhao JH. Incidence and risk of severe neutropenia in advanced cancer patients treated with cetuximab: a meta-analysis. Drugs R D 2011; 11 (4): 317-26.

68. Food and Drug Administration USFaD. 07/23/2018. p. https://fis.fda.gov/.

69. Nahid P, Dorman SE, Alipanah N, Barry PM, Brozek JL, Cattamanchi A, et al. Official American Thoracic Society/Centers for Disease Control and Prevention/ Infectious Diseases Society of America Clinical Practice Guidelines: Treatment of Drug-Susceptible Tuberculosis. Clin Infect Dis 2016; 63 (7): e147-e95. 
70. Bonovas S, Minozzi S, Lytras T, González-Lorenzo M, Pecoraro V, Colombo S, et al. Risk of malignancies using anti-TNF agents in rheumatoid arthritis, psoriatic arthritis, and ankylosing spondylitis: a systematic review and meta-analysis. Expert Opin Drug Saf 2016;
15 (sup1): 35-54.

71. Nisar MK, Rafiq A, Östör AJ. Biologic therapy for inflammatory arthritis and latent tuberculosis: real world experience from a high prevalence area in the United Kingdom. Clin Rheumatol 2015; 34 (12): 2141-5. 